\title{
Expression of Defective Hepatitis B Virus Particles Derived from Singly Spliced RNA Is Related to Liver Disease
}

\author{
Patrick Soussan, ${ }^{1,2,3,4,7}$ Jonathan Pol, ${ }^{1,2,3}$ Florianne Garreau, ${ }^{1,2,3}$ Veronique Schneider, ${ }^{4,7}$ Catherine Le Pendeven, ${ }^{4,7}$ \\ Bertrand Nalpas, ${ }^{5,9}$ Karine Lacombe, ${ }^{4,10}$ Philippe Bonnard, ${ }^{8}$ Stanislas Pol, ${ }^{1,5,6,9}$ and Dina Kremsdorf ${ }^{1,2,3}$ \\ 'Pathogenèse des Hépatites Virales B et Immunothérapie, Institut National de la Santé et de la Recherche Médicale (INSERM) U845, \\ 2Département de Virologie, Institut Pasteur, ${ }^{3}$ Faculté de Médecine René Descartes, Université Paris 5, 'Université Pierre et Marie Curie, EA3500, \\ ${ }^{5}$ Département d'Immunologie, Institut Cochin, and ${ }^{6}$ INSERM U567 and 'Laboratoire de Virologie and ${ }^{8}$ Services des Maladies Infectieuses et \\ Tropicales, Hôpital Tenon, ${ }^{9}$ Service d'Hépatologie, Hôpital Cochin, and ${ }^{10}$ Service de Maladie Infectieuse, Hôpital Saint Antoine, Assistance \\ Publique-Höpitaux de Paris, Paris, France
}

Background. Defective hepatitis B virus (HBV) particles, generated from singly spliced HBV RNA, have been detected in chronic carriers of HBV. The present study was designed to quantify the expression of defective HBV (dHBV) and wild-type HBV (wtHBV) genomes in the serum of patients with HBV infection and its relation to the severity of liver disease.

Methods. HBV and dHBV loads were determined by quantitative polymerase chain reaction in the serum of 89 untreated HBV-infected patients (31 coinfected with human immunodeficiency virus [HIV] type 1) with liver disease of different stages. The ratio of dHBV DNA to total (wtHBV plus dHBV) HBV DNA (dHBV/HBV ratio) was used to express data independently of the level of viral replication.

Results. Despite a global correlation between $\mathrm{dHBV}$ and wtHBV load, the $\mathrm{dHBV} / \mathrm{HBV}$ ratio ranged from $0.001 \%$ to $69 \%$. The variation in $\mathrm{dHBV} / \mathrm{HBV}$ ratio was independent of HIV coinfection, HBV genotype, and precore mutations. The mean $\mathrm{dHBV} / \mathrm{HBV}$ ratio was higher in patients with severe liver necrosis and fibrosis.

Conclusions. Our data indicate that an elevated $\mathrm{dHBV} / \mathrm{HBV}$ ratio is associated with liver necroinflammation and fibrosis disease, suggesting a regulation of $\mathrm{dHBV}$ expression according to the severity of the liver disease. The dHBV/ HBV ratio may help to better define liver disease stage during HBV infection.

Persistent human hepatitis B virus (HBV) infection is a major public health problem. The major complications of chronic HBV infection are the development of liver cirrhosis and hepatocellular carcinoma (HCC) [1-3]. Evidence indicating a direct involvement of $\mathrm{HBV}$ in this process is now available [3]; indeed, recent data have demonstrated that serum HBV DNA levels are strong predictors of the risk of HCC, independent of hepatitis B e antigen (HBeAg) expression, serum alanine amino-

Received 31 August 2007; accepted 12 February 2008; electronically published 4 June 2008

Potential conflicts of interest: none reported.

Financial support: Institut National de la Santé et de la Recherche Médicale; Agence Nationale de Recherche sur le SIDA et les Hépatites Virales Igrant A020042 to the study); French Research Ministry (grant to J.P.).

Reprints or correspondence: Dr. Patrick Soussan, INSERM U845, CHU Necker, 156 rue de Vaugirard 75015, Paris, France (soussan@necker.fr).

The Journal of Infectious Diseases 2008; 198:218-25

(C) 2008 by the Infectious Diseases Society of America. All rights reserved. 0022-1899/2008/19802-0010\$15.00

DOI: $10.1086 / 589623$ transferase (ALT) level, and liver cirrhosis [4]. Moreover, retrospective studies have shown that HBV genotype and HBeAg expression may also increase the risk of HCC $[5,6]$. In addition, the effect of HBV genetic variability, including precore (preC) mutants and viral genotype, has been implicated in liver disease progression [7]. Furthermore, HIV-1 infection in HBV-infected patients increases the level of HBV DNA and the risk of cirrhosis [8]. HBV should therefore be considered as having synergistic effects with chronic inflammation in the pathogenesis of liver disease.

$\mathrm{HBV}$ is a small, enveloped DNA virus and is a member of the hepadnavirus family; its genome consists of a relaxed, circular, partially double-stranded 3.2-kb DNA molecule [9]. Five unspliced messenger (mRNA) transcripts of 3.5 (short and long), 2.4, 2.1, and $0.8 \mathrm{~kb}$ are synthesized from the HBV genome and encode for the capsid, envelope, polymerase, and transactivator $\mathrm{X}$ viral proteins [9]. The 3.5-kb short pregenomic mRNA is also involved in the HBV replication cycle via its reverse tran- 
Table 1. Clinicopathological data on patients with chronic hepatitis B virus (HBV) infection ( $n=89$ ).

\begin{tabular}{lcc}
\hline Parameter & HBV monoinfection & HBV/HIV coinfection \\
\hline Sex, no. male/female & $43 / 15$ & $29 / 2$ \\
Age, years & $34 \pm 10(19-81)$ & $36 \pm 5(22-50)$ \\
HIV load, copies/mL & $\ldots$ & $8.8 \times 10^{4} \pm 5.1 \times 10^{4}(<500-323,500)$ \\
CD4 cell count, cells/mm ${ }^{3}$ & $\ldots$ & $428 \pm 250(45-856)$ \\
Alanine aminotransferase level, IU/L & $107 \pm 15(17-610)$ & $74 \pm 14(16-374)$ \\
METAVIR histological score, no. of patients & & 6 \\
Liver necroinflammation & 28 & 13 \\
$\quad$ A0-A1 & 30 & 21 \\
$\quad$ A2-A3 & & 10 \\
Liver fibrosis & 27 & \\
$\quad$ F0-F2 & 31 & \\
F3-F4 & & \\
\hline
\end{tabular}

NOTE. Data are means \pm SEs (ranges), unless otherwise specified.

scription, which occurs in core particles [9]. In addition to unspliced HBV mRNA, singly and doubly spliced 2.2-kb mRNAs arising from $3.5-\mathrm{kb}$ pregenomic mRNA have been identified in HBV DNA-transfected cell lines and in HBV-infected liver [1016]. The major spliced HBV mRNA that lacks intron 2447/489 accounts for up to $30 \%$ of pregenomic transcripts, as revealed in transfected cells and in the livers of chronically infected patients [10-13]. The mechanism for the regulation of splicing is still not clearly understood. Unlike the retrovirus model, the influence of HBV protein expression and viral genetic variability on splicing regulation has never been investigated. However, a recent report suggested that the posttranscriptional regulatory element, which is involved in the nuclear export of intronless viral subgenomic RNAs, presents regulatory elements that control viral pregenome processing by stimulating or inhibiting splicing [17]. We and others have shown that spliced mRNA is packaged, reverse transcribed, and leads to the secretion of defective viral particles $[14,15,18-20]$. Defective viruses are maintained in the population through transcomplementation with wild-type helper virus [18]. The regulation of spliced HBV RNA packaging is not well defined; however, recent data have indicated that mutations within the $\mathrm{C}$-terminus of $\mathrm{HBV}$ core protein may influence the encapsidation of spliced HBV RNA [21, 22]. In addition, the processing of defective $\mathrm{HBV}$ ( $\mathrm{dHBV}$ ) secretion may differ from that of wild-type HBV (wtHBV) secretion [20].

Defective HBV genomes contain open reading frames that may encode for various fusion and truncated proteins. It has been reported that a polymerase and surface fusion protein encoded by spliced RNA is incorporated into viral particles and may then play a role in the viral life cycle [16]. In addition, it has also been demonstrated that the expression of spliced duck HBV RNA encoding for the large surface protein is important for replication in infected hepatocytes [23]. In woodchucks chronically infected with woodchuck hepatitis virus (WHV), 2 different spliced WHV RNAs potentially encoding for core and polymerase fusion proteins were secreted as defective particles [24, 25].

The most frequently detected dHBV genomes result from the reverse transcription of singly spliced 2.2-kb mRNAs [14]. We previously demonstrated that this spliced HBV genome leads to the in vivo expression of a new HBV splice-generated protein (HBSP), which corresponds to fusion of the $\mathrm{N}$-terminal part of polymerase and a new open reading frame created by the single splicing event [26]. HBSP expression did not modulate the level of HBV replication, but indirect in vivo detection of HBSP may be related to viral load and liver fibrosis [27].

Overall, it is clear that dHBV particles are present in the liver and serum of chronically infected patients. However, the clinical impact of their expression during liver disease remains to be established. The aim of the present study was, therefore, to quantify dHBV and wtHBV particles in serum samples and to evaluate the impact of HBV genetic variability and liver status on dHBV particle expression in untreated individuals with chronic HBV infection.

\section{METHODS}

Patients and samples. Eighty-nine chronic HBV carriers who were positive for hepatitis $B$ surface antigen, had a detectable HBV load, were negative for markers of hepatitis $C$ and $D$ virus, and had never received anti-HBV therapy were randomly included in our study (table 1). Untreated patients were selected to avoid any bias resulting from HBV fitness due to antiviral treatment. Of the selected patients, 31 were coinfected with HIV-1. HIV coinfection is usually associated with rapid progression to liver cirrhosis [8]. ALT activity and HBeAg levels were evaluated by standard biological assays (bioMérieux). For HBV/HIV coinfected patients, CD4 cell count (Coulter) and HIV load (Bayer) were quantified. Liver biopsy specimens were obtained during a 3-month period around the date of serum sample, em- 

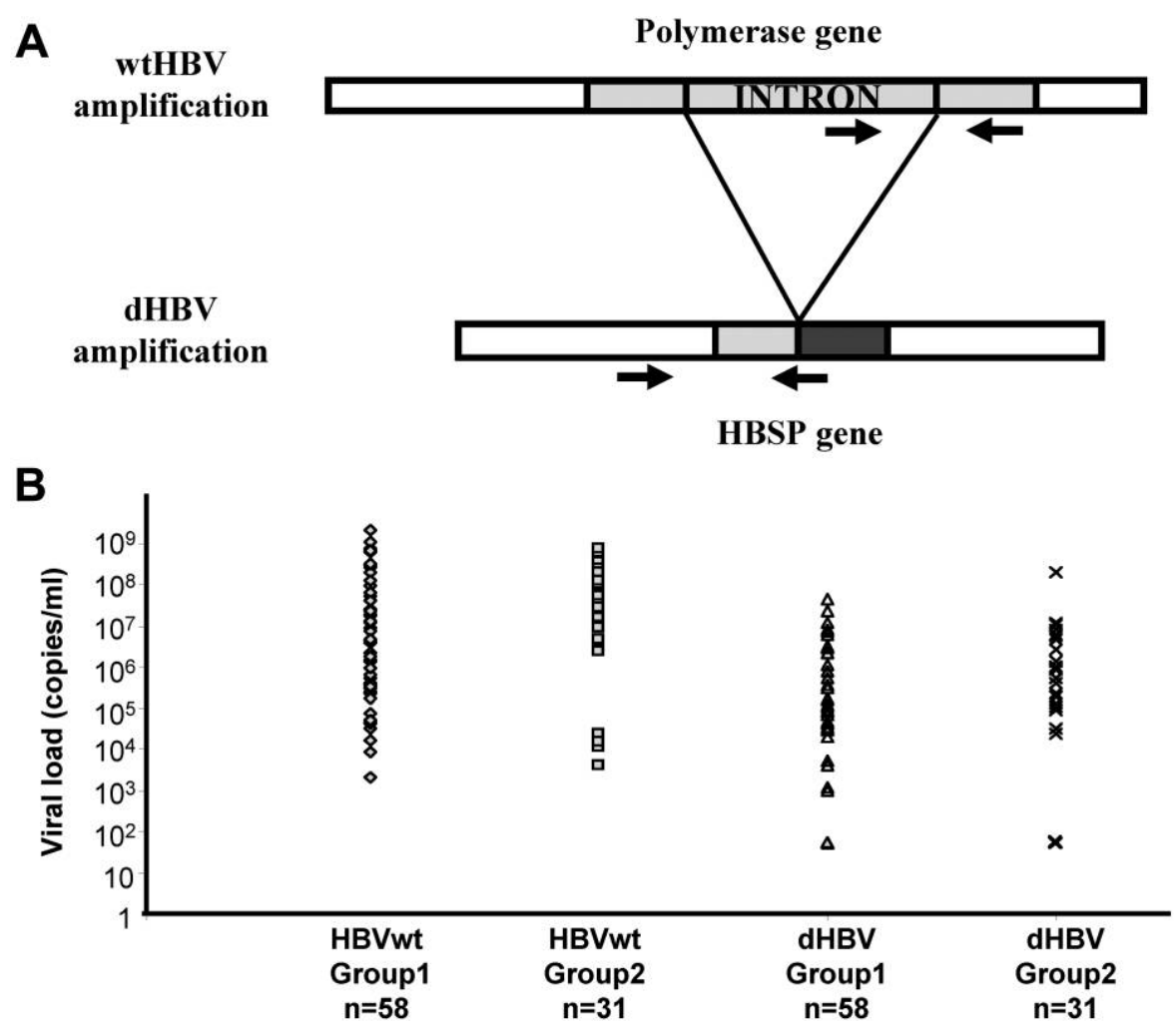

Figure 1. A, Schematic representation of wild-type hepatitis B virus (wtHBV) DNA and defective HBV (dHBV) DNA generated from singly spliced 2.2-kb HBV pregenomic RNA. For wtHBV, the gene encoding for polymerase is shown in light gray; for dHBV, the HBV splice-generated protein (HBSP) gene, in frame with polymerase, is shown in light gray, whereas the 3 ' part encoding for the original sequence is shown in dark gray. Arrows indicate the location of primers for specific amplification of either wtHBV or dHBV DNA. B, wtHBV and dHBV loads in patient groups 1 (HBV monoinfection) and 2 (HBV/HIV coinfection). Values for wtHBV and dHBV loads were determined by real-time quantitative polymerase chain reaction and are represented using logarithmic scales.

bedded in paraffin, and sectioned; sections were stained with hematoxylin-eosin. Liver biopsy specimens were classified as exhibiting no or moderate (A0-A1) or severe ( $\mathrm{A} 2-\mathrm{A} 3)$ liver necrosis and no or moderate (F0-F2) or severe (F3-F4) liver fibrosis, using the METAVIR scoring method. For 12 patients with HIV-1 coinfection, the fibrosis score was evaluated by highresolution real-time ultrasound on the basis of a quantitative scoring system. Necroinflammation scores were not available for these 12 patients.

Real-time polymerase chain reaction (PCR). HBV DNA was extracted from serum samples $(200 \mu \mathrm{L})$ by means of a spin column (Qiagen) and eluted in $200 \mu \mathrm{L}$ of water. Real-time quantitative PCR was performed using the LightCycler system (Roche) in $20 \mu \mathrm{L}$ of LightCycler DNA Master SYBR Green Mix (Roche) containing $3 \mathrm{mmol} / \mathrm{L} \mathrm{MgCl}_{2}$ and $2 \mu \mathrm{L}$ of viral DNA extract. The $\mathrm{dHBV}$ primers were 5'-AGTGTGGATTCGCACTCC-3' (forward; nt 2271-2289) and 5'-CTGGTTGTTGATGATCATT-3' (reverse; nt 504-489^2447-2445); the wtHBV primers, forward 5'-ATCTTCTTGTTGGTTCTTCT-3' (forward; nt 430-450) and 5'-CTGAAAGCCAAACAGTGG-3' (reverse; nt 733-715). Each PCR cycle comprised denaturation at $95^{\circ} \mathrm{C}$ for $20 \mathrm{~s}$, annealing at $65^{\circ} \mathrm{C}$ for $15 \mathrm{~s}$, and extension at $72^{\circ} \mathrm{C}$ for $12 \mathrm{~s}$ ( 45 cycles). The standard curve for quantification was calculated using serial 10-fold (500 to $10^{11}$ copies $/ \mathrm{mL}$ ) dilutions of wtHBV or dHBV plasmids.

Analysis of HBV genotypes and preC mutants. To detect mutations in the $\mathrm{HBV}$ basal core promoter (BCP) and preC regions and to determine $\mathrm{HBV}$ genotypes, a 644-bp fragment including the entire BCP (nt 1742-1849) and preC (nt 1814-1900) regions was amplified using the primers 5'-CATGGACCACCGTGAAC-3' (forward; nt 1605-1621) and 5'-AGTGCGAATCCACACTC-3' (reverse; nt 2284-2268) and was sequenced in an Applied Biosystems 3100 automatic sequencer. In the BCP region, mutations in $\mathrm{nt}$ 1762-1764 were analyzed; in the preC region, all nucleotide changes were analyzed. Viral genotypes were established by aligning the $\mathrm{preC} / \mathrm{core}(\mathrm{preC} / \mathrm{C})$ sequences obtained with consensus sequences of HBV genotypes A, B, C, D, E, F, and G from GenBank.

Statistical analysis. Statistical analysis was performed using the nonparametric Mann-Whitney $U$ test; results are given as means \pm SEs and medians. The correlation assay was evaluated by the Spearman rank correlation test (version 5.0; StatView software) (SAS). Analysis of variance was performed to compare HBV genotype. Differences were considered significant at $P<.05$. The Bonferroni correction was used to determine the $P$ value cutoff for liver status (ALT activity, liver necroinflammation, and fibrosis). 


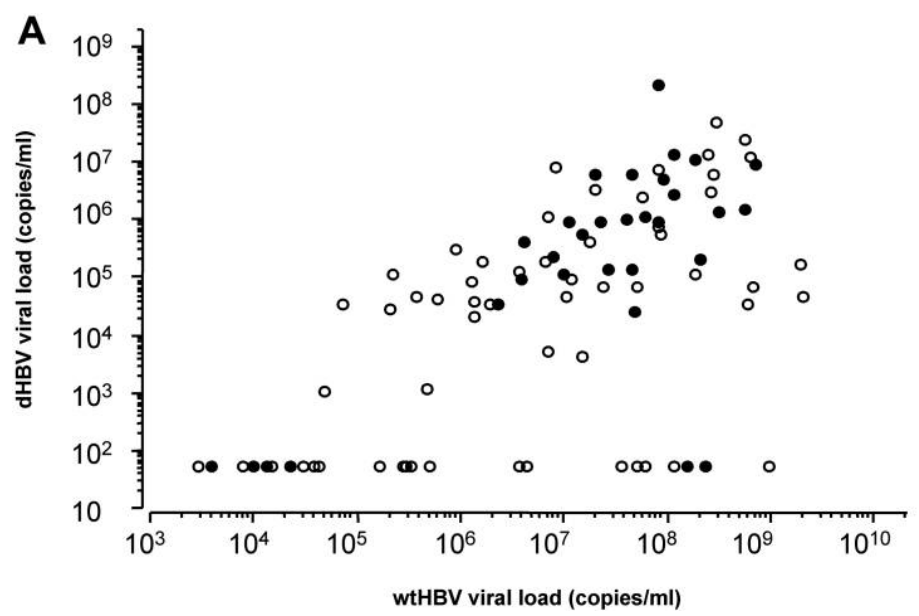

B

Figure 2. A, Relationship between the level of defective hepatitis B virus (dHBV) DNA and that of wild-type HBV (wtHBV) DNA in serum samples from 89 chronic HBV carriers. Black circles correspond to HBV/HIV-coinfected patients (group 2), and white circles correspond to HBV-monoinfected patients (group 1). Values for wtHBV and dHBV loads were determined by real-time quantitative polymerase chain reaction and are represented using logarithmic scales. When dHBV DNA was undetectable, an arbitrary value of 50 copies $/ \mathrm{mL}$ (10-fold below the threshold of detection) was attributed. B, Box plot of the distribution of the $\mathrm{dHBV} / \mathrm{HBV}$ ratios (i.e., the ratio of dHBV DNA to total [wtHBV plus dHBV $\{w t+d\}$ ] HBV DNA) in groups 1 and 2. Ratios are expressed as percentages.

\section{RESULTS}

Quantification of $\mathrm{dHBV}$ and $w \mathrm{HBV}$ DNA in the serum of chronic $\mathbf{H B V}$ carriers. $\mathrm{dHBV}$ and wtHBV DNA were quantified by real-time PCR in the serum samples of patients either infected with $\mathrm{HBV}$ alone (group $1 ; n=58$ ) or coinfected with HBV and HIV-1 (group 2; $n=31$ ) (table 1). To quantify wtHBV DNA specifically, one of the primers was located inside the intronic sequence, whereas, for dHBV DNA amplification, one of the primers encompassed splice donor and acceptor sites (figure $1 A$ ). The set of primers for $\mathrm{dHBV}$ was unable to amplify wtHBV DNA plasmid, thus demonstrating the specificity of these primers (data not shown). All samples tested were positive for wtHBV DNA, with mean \pm SE viral loads of $1.7 \times 10^{8} \pm 0.5 \times 10^{8}$ and $1.1 \times 10^{8} \pm 0.3 \times 10^{8}$ copies $/ \mathrm{mL}$ in groups 1 and 2, respectively (figure $1 B$ ). The level of dHBV DNA was significantly lower than that of wtHBV DNA in both groups. Defective viral DNA was detected in $73 \%$ of samples (viral load $\geqslant 500$ copies $/ \mathrm{mL}$ ), with means \pm SEs of $3.1 \times 10^{6} \pm 1.3 \times 10^{6}$ and $10 \times 10^{6} \pm 7.9 \times 10^{6}$ copies/ $\mathrm{mL}$ in groups 1 and 2 , respectively (figure $1 B$ ).

Both $\mathrm{dHBV}$ and wtHBV DNA are generated from reverse transcription of $\mathrm{HBV}$ pregenomic mRNA, either unspliced or singly spliced [10-16]. Thus, as expected, a correlation was observed between the levels of $\mathrm{dHBV}$ and wtHBV DNA expression for all patients $(r=0.57 ; P<.0001)$ and in groups 1 $(r=0.55 ; P<.0001)$ and $2(r=0.54 ; P=.003)$ (figure $2 A)$. Strikingly, despite this correlation, defective particles were detected in 2 of 12 patients with low replication (wtHBV load $<1 \times 10^{5}$ copies $/ \mathrm{mL}$ ). In addition, in 14 of 77 patients with high replication ( $w$ tHBV load $>1 \times 10^{5}$ copies $/ \mathrm{mL}$ ), no dHBV particles were detected. These data suggest that $\mathrm{dHBV}$ expression is related to more than just the level of $\mathrm{HBV}$ replication and raise the question of the relationship between the regulation of $\mathrm{HBV}$ splicing and viral liver disease.

Ratio of $d H B V$ to total $H B V$ particles in the serum of chronic HBV carriers. To study dHBV expression independent of viral replication, the ratio of $\mathrm{dHBV}$ DNA to total (wtHBV plus dHBV) HBV DNA (dHBV/HBV ratio) was compared for each patient. To take into account patients with undetectable dHBV DNA, an arbitrary value of 50 copies/mL (10-fold below the threshold of detection) was attributed. The dHBV/ HBV ratios were similar in both patient groups (for group 1, mean $\pm \mathrm{SE}$ of $4.8 \% \pm 1.2 \%$, range of $0.001 \%-46 \%$ and median of $0.8 \%$; for group 2 , mean \pm SE of $5.1 \% \pm 2.3 \%$, range of $0.001 \%-69 \%$, and median of $1.3 \% ; P=.29$ ) (figure $2 B$ ). The absence of a difference between the groups suggested that HIV-1 infection has no impact on HBV splicing events and the formation of defective particles. Thus, both HBV and HBV/HIV groups were pooled to explore whether the variations observed in $\mathrm{dHBV} / \mathrm{HBV}$ ratios (from $0.001 \%$ to $69 \%$ ) might be related to HBV genetic status or liver disease.

The $\mathrm{dHBV} / \mathrm{HBV}$ ratio and $\mathrm{HBV}$ status. HBV genotype and mutations in the preC/C region have been implicated in the modulation of viral replication and $\mathrm{HBeAg}$ expression $[7,28$, 29]. Thus, we wanted to investigate whether viral genotype, mutations in the preC/C region, and the level of $\mathrm{HBeAg}$ expression were involved in the variation in the $\mathrm{dHBV} / \mathrm{HBV}$ ratio. In our cohort, $86 \%$ of the HBV samples belonged to HBV genotype A, C, or E (figure $3 A$ ). Statistical analysis did not demonstrate a difference when the $\mathrm{dHBV} / \mathrm{HBV}$ ratio and $\mathrm{HBV}$ genotypes were analyzed (figure $3 A$ ). For the preC G1896A stop codon and the BCP A1762T and G1764A mutations, no significant difference 

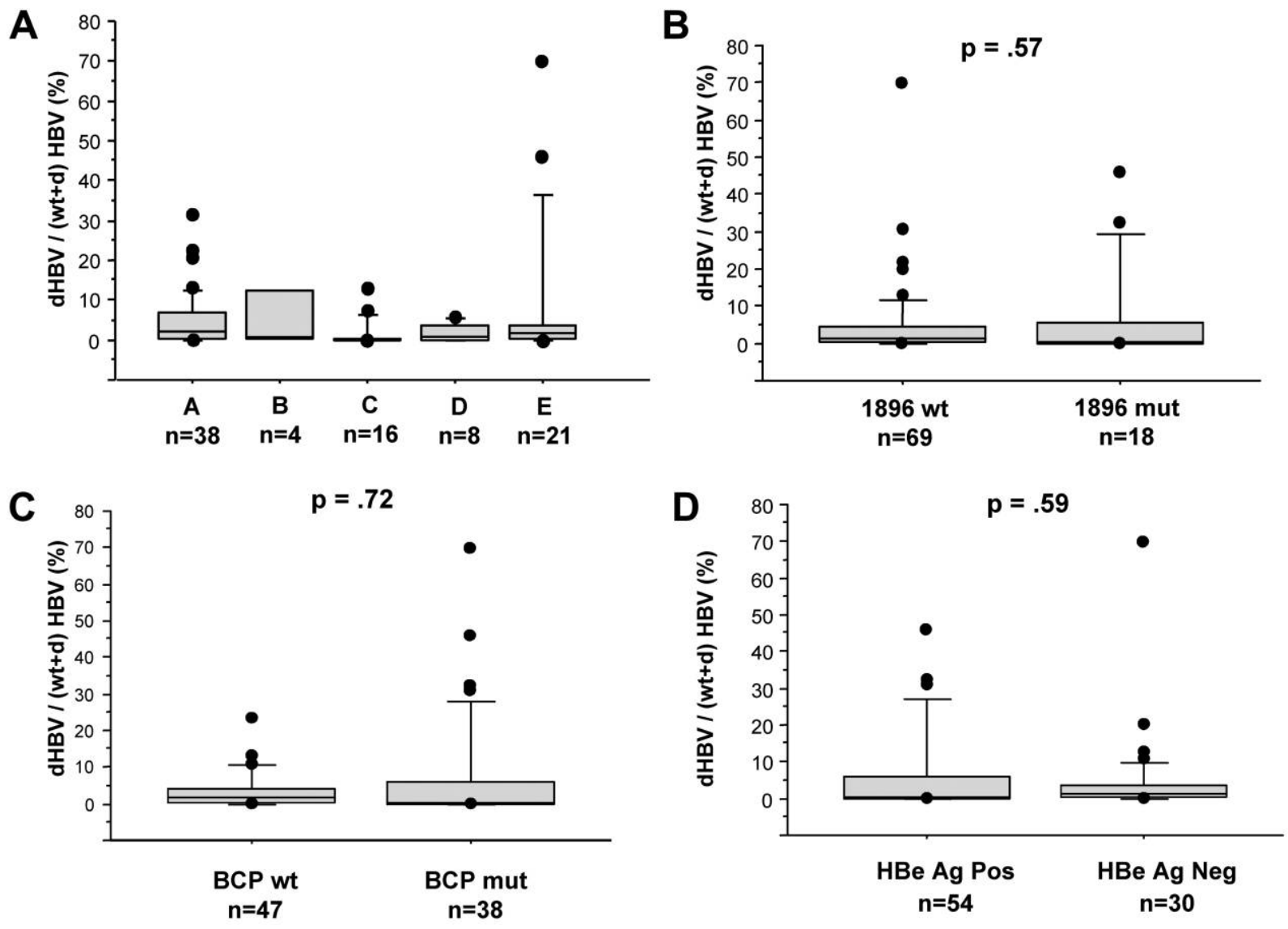

Figure 3. $d H B V / H B V$ ratios (i.e., the ratio of defective hepatitis B virus [dHBV] DNA to total [wild-type HBV plus dHBV $\{w t+d\}$ ] HBV DNA) according to viral status. Ratios are expressed as percentages. Panel A shows a box plot of dHBV/HBV ratios according to HBV genotype. HBV genotypes (A, B, C, D, and E) were determined for 87 of 89 chronic HBV carriers on the basis of sequence analysis of the capsid gene. In our cohort, $86 \%$ of HBV samples belonged to HBV genotypes $A, C$, or E. No significant difference was found between genotypes $A, C$, and $E$ by analysis of variance. For HBV genotypes $B$ and $D$, recruitment was insufficient to allow for statistical analysis. Box plots are also shown for dHBV/HBV ratios as functions of the G1896A mutation (mut) in the precore/core sequence (B), of the basal core promoter (BCP) A1762T and G1764A mutations (C), and of hepatitis B e antigen (HBeAg) expression (D). Statistical analysis was performed with the nonparametric Mann-Whitney $U$ test. Neg, negative; Pos, positive.

was observed when the $\mathrm{dHBV} / \mathrm{HBV}$ ratios were compared (figure $3 B$ and $3 C$ ). Finally, the $\mathrm{dHBV} / \mathrm{HBV}$ ratio was not significantly different between HBeAg-positive (mean \pm SE, $4.0 \% \pm 1.3 \%$; median, $1.3 \%$ ) and HBeAg-negative (mean \pm SE, $6.2 \% \pm 2.1 \%$; median, $0.5 \%)$ patients $(P=.59$ ) (figure $3 D)$. These data suggest that the $\mathrm{dHBV} / \mathrm{HBV}$ ratio is not influenced by mutations usually involved in the modulation of $\mathrm{HBV}$ replication.

The $\mathrm{dHBV/HBV}$ ratio and liver disease. The next step was to study the relationship between $\mathrm{HBV}$-associated liver diseases and the $\mathrm{dHBV} / \mathrm{HBV}$ ratio. No significant difference could be seen in the $\mathrm{dHBV} / \mathrm{HBV}$ ratio according to ALT activity (for ALT level less than twice the normal value, mean \pm SE of $3.2 \% \pm 0.7 \%$ and median of $0.7 \%$; for ALT level more than or equal to twice the normal value, mean \pm SE of $5.7 \% \pm 2.4 \%$ and median of $1.4 \%$; $P=.71$ ) (figure $4 A$ ). However, $\mathrm{dHBV} / \mathrm{HBV}$ ratios were significantly higher in patients with severe fibrosis (mean $\pm \mathrm{SE}$, $8.1 \% \pm 2.3 \%$; median, $1.8 \%$ ) than in those with moderate fi- brosis (mean $\pm \mathrm{SE}, 2.2 \% \pm 0.5 \%$; median, $0.9 \%)(P=.04)$ (figure $4 B$ ). Furthermore, dHBV/HBV ratios were significantly higher in the population with severe liver necrosis (mean $\pm \mathrm{SE}$, $7.6 \% \pm 2.1 \%$; median, $1.8 \%$ ) than in those with moderate or no liver necrosis (mean $\pm \mathrm{SE}, 1.8 \% \pm 0.6 \%$; median, $0.3 \%$ ) $(P=.009)$ (figure $4 C)$. The 4 -fold increase in mean $\mathrm{dHBV} /$ $\mathrm{HBV}$ ratio in patients with severe liver necrosis was a consequence of a 2-fold decrease in wtHBV load and a 2-fold increase in $\mathrm{dHBV}$ load. However, no significant difference was observed between patients with moderate or no liver necrosis and those with severe liver necrosis for either wtHBV load (mean \pm SE, $2.4 \times 10^{8} \pm 0.9 \times 10^{8}$ and $0.9 \times 10^{8} \pm 0.3 \times 10^{8}$ copies $/ \mathrm{mL}$, respectively) or $\mathrm{dHBV}$ load (mean $\pm \mathrm{SE}$, $2.5 \times 10^{6} \pm 1.5 \times 10^{6}$ and $6.2 \times 10^{6} \pm 4.6 \times 10^{6}$ copies/ $\mathrm{mL})(P=.64)$. Similar results were observed for fibrosis (data not shown). Thus, our data support the notion of a specific regulation of $\mathrm{dHBV}$ expression according to the severity of liver disease. 


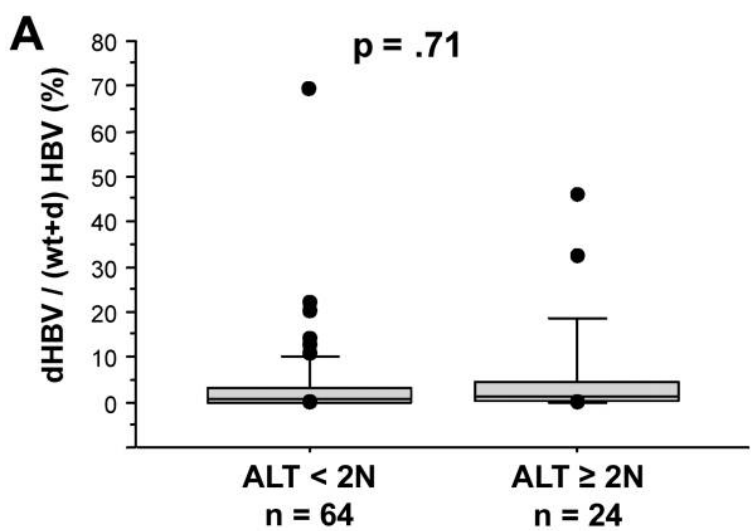

B
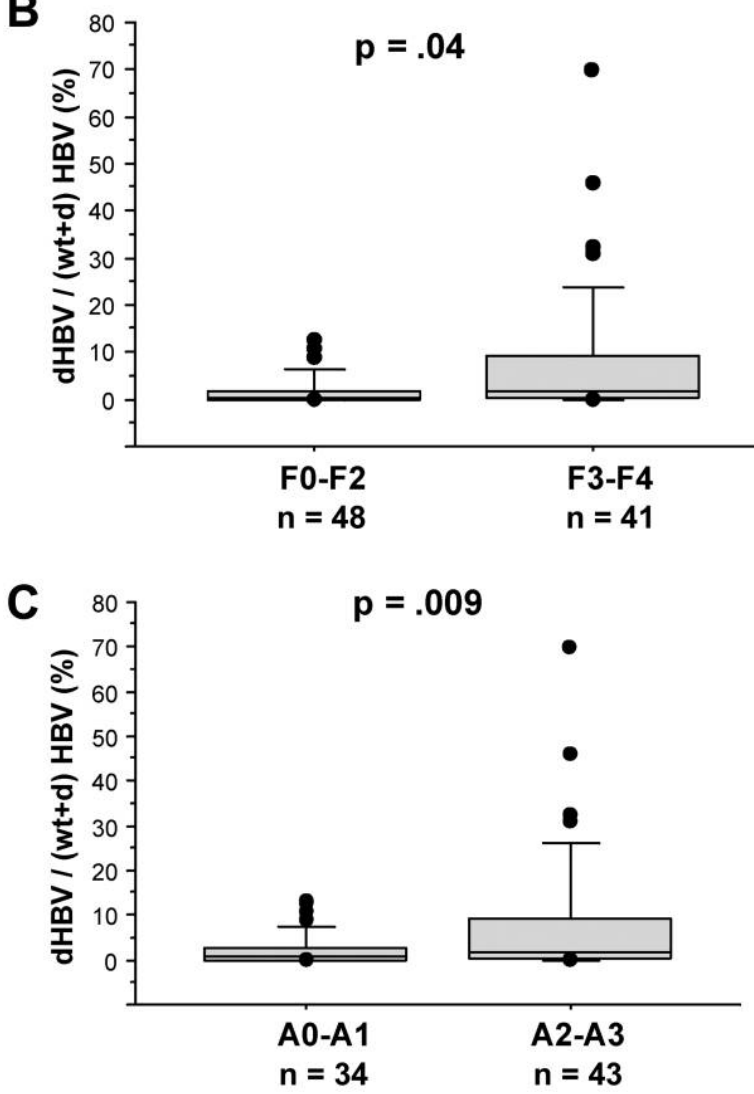

Figure 4. $\mathrm{dHBV} / \mathrm{HBV}$ ratios (i.e., the ratio of defective hepatitis $B$ virus [dHBV] DNA to total [wild-type HBV plus dHBV $\{w t+d\}]$ HBV DNA) according to liver disease status. Ratios are expressed as percentages. Shown are box plots of dHBV/HBV ratios as a function of alanine aminotransferase (ALT) activity (A), of liver fibrosis status (B), and of liver necroinflammatory histological status $(C)$. ALT activity was considered positive when the ALT level was at least twice the normal value (2N). Liver biopsy specimens were classified as having no or moderate (A0-A1) or severe (A2-A3) liver necrosis and no or moderate (F0-F2) or severe (F3-F4) liver fibrosis, using the METAVIR scoring system. Statistical analysis was performed with the nonparametric Mann-Whitney $U$ test.

\section{DISCUSSION}

The present study was performed to investigate the regulation of dHBV particle expression in HBV-related liver disease. Indeed, although a study using nonquantitative methods showed a de- crease in the ratio of spliced to unspliced RNA in patients with high versus low histologic activity indexes [30], regulation of dHBV particle expression remained unclear. Thus, levels of wtHBV and $\mathrm{dHBV}$ genomes were quantified in serum samples from patients with chronic $\mathrm{HBV}$ infection with or without HIV-1 coinfection. In patients with active HBV replication (wtHBV $>1 \times 10^{5}$ copies $/ \mathrm{mL} ; 86 \%$ of studied patients), the dHBV load was $>1 \times 10^{5}$ copies $/ \mathrm{mL}$ in $56 \%$ of cases. This result raises the question of the function of splicing events and the formation of defective particles in the natural history of HBV infection.

The $\mathrm{dHBV} / \mathrm{HBV}$ ratio is a measure independent of the level of viral replication. The percentage of $\mathrm{dHBV}$ particles varied from $0.001 \%$ to $69 \%$ of the total viral circulating population. Clearly, for most patients, the circulating amount of $\mathrm{dHBV}$ particles was low in comparison with wtHBV particles. The mean dHBV/ HBV ratio was far from what was expected, considering the reported intracellular proportion of spliced pregenomic RNA ( $\sim 30 \%$ of HBV pregenomic mRNA) [10-13]. This difference might be explained, as reported elsewhere, by the intracellular retention of defective particles compared with wild-type virus secretion [20]. Altogether, despite a correlation between $\mathrm{dHBV}$ and wtHBV particles, dHBV expression might depend on more than the HBV replication level. The $\mathrm{dHBV} / \mathrm{HBV}$ ratio was not influenced by HIV-1 coinfection, HBV genotype, or mutations in the basal core or preC regions. In contrast, we found that the $\mathrm{dHBV} / \mathrm{HBV}$ ratio was significantly elevated in serum samples obtained from patients with severe liver fibrosis or necrosis. However, the difference in the $\mathrm{dHBV} / \mathrm{HBV}$ ratio regarding fibrosis is at the limit of statistical significance $(P=.04)$ and is not significant after the Bonferroni correction (threshold of $P=.016)$. This might reflect the inclusion of HIV-coinfected patients in our cohort. Indeed, it has been reported previously that HIV infection influenced the progression of liver fibrosis without increasing liver necroinflammation in HBV chronic carriers [8]. When HIV-coinfected patients were excluded from the analysis, the relationship between the $\mathrm{dHBV} / \mathrm{HBV}$ ratio and liver fibrosis was more notable (median, $1.8 \%$ vs. $0.1 \% ; P=.02$ for the difference, which is the limit of significance using the Bonferroni correction). Nevertheless, these data suggest a direct association between the $\mathrm{dHBV} / \mathrm{HBV}$ ratio and the severity of liver disease.

A lack of correlation between liver histology and single measurements of HBV DNA level has been reported previously [31]. However, effective suppression of viral replication in patients with chronic HBV infection has been shown to slow disease progression and improve patient outcomes [32]. Furthermore, loss of viral control in patients infected with HBV strains resistant to antiviral treatment leads to more rapid disease progression [33]. These data illustrate that serum HBV DNA level is a predictive factor for cirrhosis and HCC. In addition, a recent report showed that, in untreated chronic HBV carriers, the risk of cir- 
rhosis increased with increasing HBV DNA level, independent of serum ALT level or HBeAg status [34].

Taken together, our data reveal a correlation between $\mathrm{dHBV}$ expression and HBV replication capacity but also indicate a specific regulation of $\mathrm{dHBV}$ expression that is related to the severity of liver disease. In this context, reports have highlighted the modulation of splicing events in cellular gene expression during the progression of liver disease $[35,36]$. In addition, it has recently been suggested that HBV RNA splicing could be modulated by the splicing factor PSF [17]. Consequently, it could be hypothesized that the expression of defective particles is modulated by the extracellular environment of hepatocytes during liver disease.

During the course of HBV infection, the mechanisms of liver injury are usually attributed to the host immune response. However, evidence now suggests that expression of certain HBV proteins has a direct impact on liver disease [3]. Our data show that the regulation of $\mathrm{dHBV}$ particles is linked to the severity of viral liver disease. These defective particles may be involved in liver disease through either the modulation of viral protein expression or the expression of spliced specific viral protein. Indeed, we have shown previously that singly spliced HBV RNA and its related defective particles allowed overexpression of HBV core antigen and generated the expression of HBSP [26, 37]. We have also demonstrated previously that HBSP is involved in the modulation of cell viability [26]. Further studies are required to determine the direct involvement of HBSP in the pathogenesis of liver disease.

The monitoring of HBV-related liver disease remains a major challenge in clinical practice. Recent reports have demonstrated that liver necroinflammation and fibrosis can be identified by noninvasive tests using different combinations of blood markers $[38,39]$. The present study highlights the possibility that the $\mathrm{dHBV} / \mathrm{HBV}$ ratio might be modulated during liver disease. However, the $\mathrm{dHBV} / \mathrm{HBV}$ ratio must be investigated in a follow-up study to evaluate the modulation of this ratio during the progression of liver disease. Finally, a prospective clinical study will make it possible to evaluate whether this ratio can be considered, along with biological and virological parameters, to help define the clinical stages of liver disease during HBV infection.

\section{Acknowledgments}

We thank J. B. Trabut for collecting clinical data and J. P. Jais and M. Weiss for critical reading of the manuscript.

\section{References}

1. Chisari FV, Ferrari C. Hepatitis B virus immunopathogenesis. Annu Rev Immunol 1995; 13:29-60.

2. Villeneuve JP. The natural history of chronic hepatitis B virus infection. J Clin Virol 2005; 34(Suppl 1):S139-42.
3. Kremsdorf D, Soussan P, Paterlini-Brechot P, Brechot C. Hepatitis B virus-related hepatocellular carcinoma: paradigms for viral-related human carcinogenesis. Oncogene 2006; 25:3823-33.

4. Chen CJ, Yang HI, Su J, et al. Risk of hepatocellular carcinoma across a biological gradient of serum hepatitis B virus DNA level. JAMA 2006; 295:65-73.

5. Orito E, Mizokami M. Hepatitis B virus genotypes and hepatocellular carcinoma in Japan. Intervirology 2003; 46:408-12.

6. Yang HI, Lu SN, Liaw YF, et al. Hepatitis B e antigen and the risk of hepatocellular carcinoma. N Engl J Med 2002; 347:168-74.

7. Buti M, Rodriguez-Frias F, Jardi R, Esteban R. Hepatitis B virus genome variability and disease progression: the impact of pre-core mutants and HBV genotypes. J Clin Virol 2005; 34(Suppl 1):S79-82.

8. Colin JF, Cazals-Hatem D, Loriot MA, et al. Influence of human immunodeficiency virus infection on chronic hepatitis B in homosexual men. Hepatology 1999; 29:1306-10.

9. Seeger C, Mason WS. Hepatitis B virus biology. Microbiol Mol Biol Rev 2000; 64:51-68.

10. Wu H-L, Chen P-J, Tu S-J, Lin M-H, Lai M-Y, Chen D-S. Characterization and genetic analysis of alternatively spliced transcripts of hepatitis $\mathrm{B}$ virus in infected human liver tissues and transfected Hep G2 cells. JVirol $1991 ; 65: 1680-6$.

11. Chen P-J, Chen C-R, Sung J-L, Chen D-S. Identification of a doubly spliced viral transcript joining the separated domains for putative protease and reverse transcriptase of hepatitis B virus. J Virol 1989; 63:4165-71.

12. Suzuki T, Kajino K, Masui N, Saito I, Miyamura T. Alternative splicing of hepatitis B virus RNAs in HepG2 cells transfected with the viral DNA. Virology 1990; 179:881-5.

13. Su TS, Lai CJ, Huang JL, et al. Hepatitis B virus transcript produced by RNA splicing. J Virol 1989; 63:4011-8.

14. Günther S, Sommer G, Iwanska A, Will H. Heterogeneity and common features of defective hepatitis B virus genomes derived from spliced pregenomic RNA. Virology 1997; 238:363-71.

15. Terré S, Petit MA, Bréchot C. Defective hepatitis B virus particles are generated by packaging and reverse transcription of spliced viral RNAs in vivo. J Virol 1991; 65:5539-43.

16. Huang HL, Jeng KS, Hu CP, Tsai CH, Lo SJ, Chang C. Identification and characterization of a structural protein of hepatitis $\mathrm{B}$ virus: a polymerase and surface fusion protein encoded by a spliced RNA. Virology 2000; 275:398-410.

17. Heise T, Sommer G, Reumann K, Meyer I, Will H, Schaal H. The hepatitis $\mathrm{B}$ virus PRE contains a splicing regulatory element. Nucleic Acids Res 2006; 34:353-63.

18. Rosmorduc O, Petit M-A, Pol S, et al. In vivo and in vitro expression of defective hepatitis $B$ virus particles generated by spliced hepatitis B virus RNA. Hepatology 1995; 22:10-19.

19. Gunther S, Li BC, Miska S, Kruger DH, Meisel H, Will H. A novel method for efficient amplification of whole hepatitis B virus genomes permits rapid functional analysis and reveals deletion mutants in immunosuppressed patients. J Virol 1995; 69:5437-44.

20. Sommer G, van Bommel F, Will H. Genotype-specific synthesis and secretion of spliced hepatitis B virus genomes in hepatoma cells. Virology 2000; 271:371-81.

21. Kock J, Nassal M, Deres K, Blum HE, von Weizsacker F. Hepatitis B virus nucleocapsids formed by carboxy-terminally mutated core proteins contain spliced viral genomes but lack full-size DNA. J Virol 2004; 78: 13812-8.

22. Le Pogam S, Chua PK, Newman M, Shih C. Exposure of RNA templates and encapsidation of spliced viral RNA are influenced by the argininerich domain of human hepatitis B virus core antigen (HBcAg 165-173). J Virol 2005; 79:1871-87.

23. Obert S, Zachmann-Brand B, Deindl E, Tucker W, Bartenschlager R, Schaller H. A spliced hepadnavirus RNA that is essential for virus replication. Embo J 1996; 15:2565-74. 
24. Ogston CW, Razman DG. Spliced RNA of woodchuck hepatitis virus. Virology 1992; 189:245-52.

25. Hantz O, Baginski I, Fourel I, Chemin I, Trepo C. Viral spliced RNA are produced, encapsidated and reverse transcribed during in vivo woodchuck hepatitis virus infection. Virology 1992; 190:193-200.

26. Soussan P, Garreau F, Zylberberg H, Ferray C, Brechot C, Kremsdorf D. In vivo expression of a new hepatitis $\mathrm{B}$ virus protein encoded by a spliced RNA. J Clin Invest 2000; 105:55-60.

27. Soussan P, Tuveri R, Nalpas B, et al. The expression of hepatitis B spliced protein (HBSP) encoded by a spliced hepatitis B virus RNA is associated with viral replication and liver fibrosis. J Hepatol 2003; 38:343-8.

28. Parekh S, Zoulim F, Ahn SH, et al. Genome replication, virion secretion, and e antigen expression of naturally occurring hepatitis $\mathrm{B}$ virus core promoter mutants. J Virol 2003; 77:6601-12.

29. Gunther S. Genetic variation in HBV infection: genotypes and mutants. J Clin Virol 2006; 36(Suppl 1):S3-11.

30. Sheen IS, Tsou YK, Lin SM, et al. Nuclear HBcAg and histology activity index as independent predictors of the expression of singly spliced HBVRNA. J Viral Hepat 2007; 14:70-4.

31. Dusheiko G, Antonakopoulos N. Current treatment of hepatitis B. Gut 2008; 57:105-24.
32. Liaw YF, Sung JJ, Chow WC, et al. Lamivudine for patients with chronic hepatitis B and advanced liver disease. N Engl J Med 2004; 351:1521-31.

33. Di Marco V, Marzano A, Lampertico P, et al. Clinical outcome of $\mathrm{HBeAg-negative} \mathrm{chronic} \mathrm{hepatitis} \mathrm{B} \mathrm{in} \mathrm{relation} \mathrm{to} \mathrm{virological} \mathrm{response} \mathrm{to}$ lamivudine. Hepatology 2004; 40:883-91.

34. Iloeje UH, Yang HI, Su J, Jen CL, You SL, Chen CJ. Predicting cirrhosis risk based on the level of circulating hepatitis B viral load. Gastroenterology 2006; 130:678-86.

35. Wang XQ, Luk JM, Leung PP, Wong BW, Stanbridge EJ, Fan ST. Alternative mRNA splicing of liver intestine-cadherin in hepatocellular carcinoma. Clin Cancer Res 2005; 11:483-9.

36. Takashima $\mathrm{H}$, Nakajima $\mathrm{T}$, Moriguchi $\mathrm{M}$, et al. In vivo expression patterns of survivin and its splicing variants in chronic liver disease and hepatocellular carcinoma. Liver Int 2005; 25:77-84.

37. Rosmorduc O, Sirma H, Soussan P, et al. Inhibition of interferoninducible MxA protein expression by hepatitis B virus capsid protein. J Gen Virol 1999; 80:1253-62.

38. Cales P, Oberti F, Michalak S, et al. A novel panel of blood markers to assess the degree of liver fibrosis. Hepatology 2005; 42:1373-81.

39. Myers RP, Tainturier MH, Ratziu V, et al. Prediction of liver histological lesions with biochemical markers in patients with chronic hepatitis B. J Hepatol 2003; 39:222-30. 